\title{
Infrarenal mycotic aortic pseudoaneurysm
}

\author{
Steven Miles ${ }^{1}$ and Maher Khazem MS ${ }^{2 *}$ \\ ${ }^{1}$ Clinical Assistant and Professor at Florida State University College of Medicine, Daytona Campus, USA \\ ${ }^{2}$ Florida State University College of Medicine, Daytona Campus, Daytona Beach, USA
}

\begin{abstract}
Aortic pseudoaneurysm is a serious vascular pathology that requires consistent monitoring and intervention in some cases. Rupture is among the most serious complications, but infection is also a big concern. The most common organisms are bacterial typically S. Aureus or Salmonella spp. Mycotic seeding of aneurysms is a less common complication but should be taken just as seriously.

Here we present a case of a 56-year-old male with HIV who presented with a pseudoaneurysm rupture that was being repaired and found to be infected with Mycobacterium Avium Complex. The case highlights a rare cause of pseudoaneurysm infection and is the first case of an infrarenal MAC infected pseudoaneurysm and advocates for further study. In addition, it emphasizes the importance of surveillance and management of pseudoaneurysms (especially in immunocompromised patients) to prevent complications.
\end{abstract}

\section{Introduction}

Aneurysms are atypical focal dilations of arteries. Secondary infection of a pre-existing aneurysm is the predominant method of infection. Mycotic aneurysms however are more often due to septic embolization or bacteremia that leads to a mycotic seeding and infection.

Mycotic aneurysms may develop anywhere in the body but have been most reported in the intracranial arteries, visceral arteries and upper and lower extremities. They typically occur at bifurcations of the arterial tree.

This is a case report of a 56-year-old HIV positive male whose initial presentation to the ED for abdominal pain led to a series of tests which incidentally found a ruptured abdominal pseudoaneurysm. The pseudoaneurysm was found to be growing Mycobacterium Avium Complex upon culture.

This is the first case to report a mycotic infrarenal aortic pseudoaneurysm caused by Mycobacterium Avium Complex.

\section{Case presentation}

A 56-year-old male with PMH of HIV, Hepatitis C, and chronic pain medication dependence presenting to the emergency department with severe abdominal pain. He also reports back pain as well as some chest discomfort. Surgical history notable for CABG 3 years ago.

Vitals: 98.3 degrees Fahrenheit, 90 beats per minute, 20 breaths per minute, 100 O2 sat, BP 231/109

Labs upon admission significant for pCO2 30, Sodium 134, Potassium 3.1, random glucose 121, Calcium 8.2. Urine toxicology was positive for cocaine.

Contrast CT of abdomen shows a $5.3 \mathrm{~cm}$ infrarenal abdominal aortic pseudoaneurysm that had ruptured. (Figures 1 and 2).

He was sent to the OR emergently for open repair. The peritoneal cavity was opened and control of the proximal infrarenal aorta was

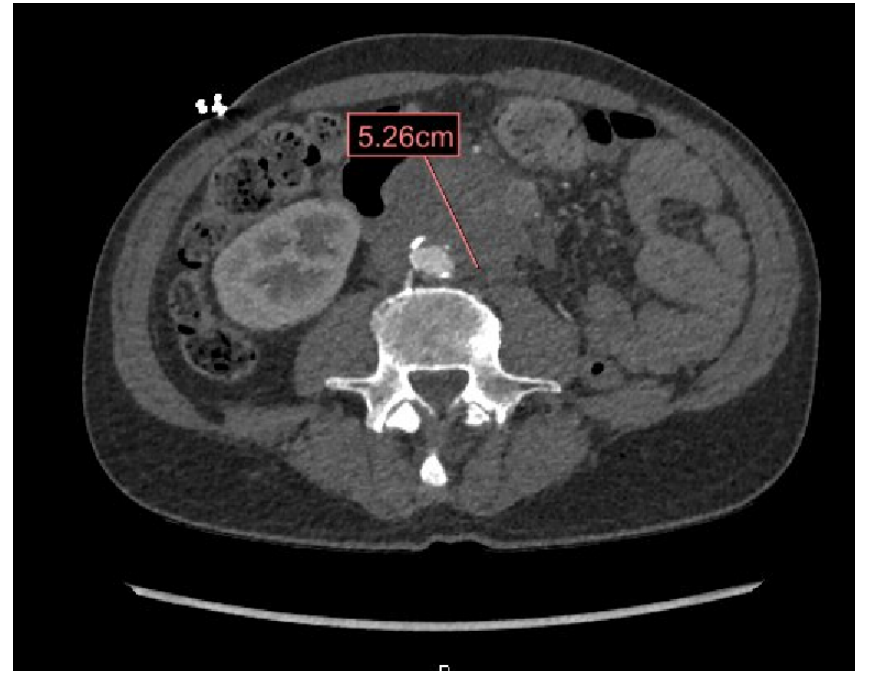

Figure 1. Axial view of $5.3 \mathrm{~cm}$ infrarenal abdominal aortic pseudoaneurysm

obtained. Upon further dissection towards the pseudoaneurysm a large phlegmon was found. The phlegmon had surrounding inflammatory processes surrounding the pseudoaneurysm sac which was highly suspicious to the vascular surgeon for an infectious etiology. The soft tissue was sent to pathology for an evaluation. Culture grew Mycobacterium Avium Complex [1,2].

The soft tissue of the pseudoaneurysm was dissected and the pseudoaneurysm sac was opened and irrigated. A $16 \mathrm{~mm}$ rifampin-

*Correspondence to: Maher Khazem, Florida State University College of Medicine, Daytona Campus, Daytona Beach, USA, E-mail: mk17h@med.fsu.edu Key words: Mycotic, pseudoaneurysm, MAC, HIV

Received: September 25, 2020; Accepted: September 29, 2020; Published: September 30, 2020 


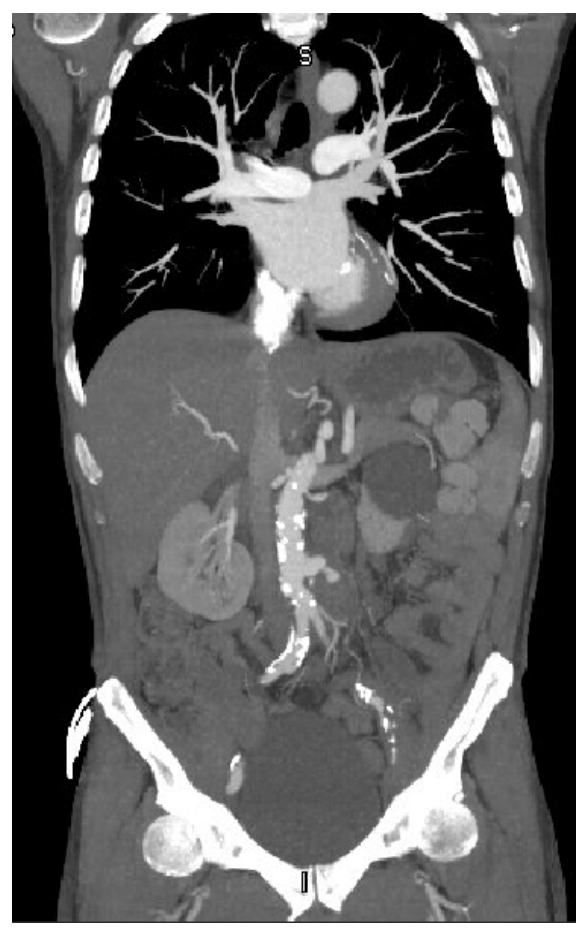

Figure 2. Coronal view of infrarenal abdominal aortic pseudoaneurysm

soaked Dacron graft was used for repair. Proximal and distal anastomosis were formed with a 3-0 Prolene suture in a running fashion. Hemostasis was achieved. The patient tolerated the procedure well [3].

Patient was placed on IV Piperacillin Tazobactam and IV Vancomycin for post-operative fevers. Patient also had post-operative anemia and was given 2 units of blood with hemoglobin remaining stable after that. His HIV CD4 count was 22 with absolute CD4 count of 112 . He was educated and counseled to continue triple antiretroviral therapy. Follow up with vascular surgery in 2 weeks $[4,5]$.

\section{Discussion}

Pseudoaneurysm complications are very serious. Mortality rates upwards of $90 \%$ for ruptured pseudoaneurysms are substantial. This is why so much emphasis goes into monitoring. More studies explaining the pathophysiology of MAC complex and other mycotic organisms are needed in relation to pseudoaneurysms and their attack on the vascular system. Mycotic seeding effects on enlargement and rupture of pseudoaneurysms is unknown. It can be inferred that mycotic infection of the vasculature contributes to instability and asymmetric growth. This is a potential future topic of research that can benefit the immunocompromised population and those who are high risk for seeding or infection of aneurysms. Potential therapies and modalities to lower risk of infection may prove to be beneficial and reduce negative outcomes in this patient population.

\section{Conclusion}

Pseudoaneurysm rupture is a life-threatening complication. Infection especially in an immunocompromised patient worsens the prognosis. This report provides an interesting case of a mycotic aneurysm in the descending aorta. This is a particularly uncommon presentation of seeding of a pseudoaneurysm by MAC complex. To date, this appears to be the first published case on a Mycobacterium Avium Complex infrarenal aortic pseudoaneurysm.

\section{References}

1. Atik FA, Navia JL, Svensson LG, Vega PR, Feng J, et al. (2006) Surgical treatment of pseudoaneurysm of the thoracic aorta. $J$ Thoracic and Cardiovas Sur132: 379-385. [Crossref]

2. Goudeketting S (2019) Endovascular aneurysm repair: Prevention and treatment of complications.

3. Montaudon M (2017) Aorta-Mycotic aneurysm. Rad Intell Assis 174: 11-15.

4. Tihan D, Aksoy M (2014) A real mycotic aneurysm-mycotic aneurysm of the abdominal aorta due to fungal infection. Turkish J Surg 30: 222-224. [Crossref]

5. Yeoh CB (1963) Tuberculous pseudoaneurysm of descending thoracic aorta. Arch Surg 86: 318. [Crossref]

Copyright: (C2020 Miles S. This is an open-access article distributed under the terms of the Creative Commons Attribution License, which permits unrestricted use, distribution, and reproduction in any medium, provided the original author and source are credited. 